\title{
Does periosteum promote chondrogenesis? A comparison of free periosteal and perichondrial grafts in the regeneration of ear cartilage
}

\begin{abstract}
Hyokyung Yoo,
Taekeun Yoon ${ }^{1}$,

Hahn-Sol Bae,

Min-Suk Kang,

Byung Jun Kim ${ }^{1}$
\end{abstract}

${ }^{1}$ Department of Plastic and

Reconstructive Surgery, Seoul National

University Hospital, Seoul; ${ }^{2}$ Idong

Public Health Center, Yongin; ${ }^{3}$ ID

Hospital, Seoul, Korea
Background: Elastic ear cartilage is a good source of tissue for support or augmentation in plastic and reconstructive surgery. However, the amount of ear cartilage is limited and excessive use of cartilage can cause deformation of the auricular framework. This animal study investigated the potential of periosteal chondrogenesis in an ear cartilage defect model.

Methods: Twelve New Zealand white rabbits were used in the present study. Four ear cartilage defects were created in both ears of each rabbit, between the central artery and marginal veins. The defects were covered with perichondrium (group 1), periosteum taken from the calvarium (group 2), or periosteum taken from the tibia (group 3). No coverage was performed in a control group (group 4). All animals were sacrificed 6 weeks later, and the ratio of neo-cartilage to defect size was measured.

Results: Significant chondrogenesis occurred only in group 1 (cartilage regeneration ratio: mean \pm standard deviation, $0.97 \pm 0.60)$, whereas the cartilage regeneration ratio was substantially lower in group $2(0.10 \pm 0.11)$, group $3(0.08 \pm 0.09)$, and group $4(0.08 \pm 0.14)(p=0.004)$. Instead of chondrogenesis, osteogenesis was observed in the periosteal graft groups. No statistically significant differences were found in the amount of osteogenesis or chondrogenesis between groups 2 and 3. Group 4 showed fibrous tissue accumulation in the defect area.

Conclusion: Periosteal grafts showed weak chondrogenic potential in an ear cartilage defect model of rabbits; instead, they exhibited osteogenesis, irrespective of their embryological origin.

Keywords: Cartilage, ear / Cartilage, elastic / Chondrogenesis / Osteogenesis

\section{INTRODUCTION}

Ear cartilage is a valuable source for tissue support or augmentation in reconstructive or cosmetic surgery. This elastic cartilage contains collagen fibers with a dense extracellular matrix. Therefore, it has solid properties, but is also sufficiently flexible to withstand repeated bending forces. Clinically, ear cartilage

\footnotetext{
Correspondence: Byung Jun Kim

Department of Plastic and Reconstructive Surgery, Seoul National University Hospital, Seoul National University College of Medicine, 101 Daehak-ro, Jongnogu, Seoul 03080, Korea

E-mail: bjkim79@gmail.com

Received September 16, 2021 / Revised October 6, 2021 / Accepted October 16, 2021
}

can be used for eyelid reconstruction, nasal tip augmentation, nasal columellar struts, or chondrocutaneous composite grafts to correct short nose deformities [1-4].

However, the amount of ear cartilage is limited, prompting the development of substitutes such as cartilage allografts or tissue-engineered cartilage. A cartilage allograft or xenograft is an attractive alternative considering the weak antigenicity and low oxygen demand of cartilage, but its high cost and foreign body reaction limit the clinical usage of this option [5-7]. Tissue engineering or biodegradable implant is another option, but the need to establish its long-term safety and efficacy remains a hurdle to be overcome before its clinical application $[8,9]$. 
Some investigators have presented successful cartilage regeneration using periosteal grafts. Most of these studies have been confined to hyaline cartilage regeneration in the knee joint using periosteum from the tibia $[10,11]$. The periosteum has chondrogenic potential if a favorable environment for chondrogenesis is provided. However, the head and neck environment differs from that of the joint space in terms of anatomical and physiological properties, as it has a relatively robust blood supply and less movement or tension.

In the present animal study, we investigated the potential of elastic ear cartilage regeneration using free periosteal grafts, and the results were compared to those of free perichondrial grafts. Furthermore, the chondrogenic potential was compared between periosteum from the calvarium and periosteum from the tibia, based on the fact that they have different embryologic origins. To the best of our knowledge, no study has directly compared the chondrogenic potential of periosteum from different embryologic origins for the regeneration of elastic cartilage.

\section{METHODS}

Twelve 12-week-old male New Zealand white rabbits weighing $2.0-2.5 \mathrm{~kg}$ were included in the present study. All procedures were performed after receiving approval from the Institutional Animal Care and Use Committee (IACUC No. 13-0164). All animals were observed for at least 1 week preoperatively to provide an accommodation period and to screen for any systemic impairment. A cocktail of $15 \mathrm{mg} / \mathrm{kg}$ Zoletil (Virbac, Carros, France) with $5 \mathrm{mg} / \mathrm{kg}$ Rompun (Bayer, Leverkusen, Germany) was administered in the quadriceps femoris muscle of the rabbits to achieve anesthesia. Intramuscular injection of cefazolin (30 mg/kg; Chong Kun Dang, Seoul, Korea) was also performed to prevent wound infection. The fur on the auricles, scalp, and right medial tibia was removed with an electric shaver. The skin was prepared to provide aseptic conditions during the procedure.

\section{Flap elevation}

Four skin flaps, each measuring $1.2 \times 1.2 \mathrm{~cm}$, were designed on the posterior aspect of both ears between the central artery and marginal veins, taking care not to interfere with the vessels. Semi-permanent markings of the flap design were added using a micro-tattoo machine (Digital-Pro, Bomtech, Seoul, Korea) (Fig. 1). Lidocaine (2\%; Huons, Seongnam, Korea) was injected over the perichondrial plane on the anterior and posterior aspects of each ear to make a clear dissection plane and to facilitate flap elevation. Epinephrine was not used to avoid vasoconstriction, which can affect flap viability and cartilage regeneration. A skin incision was made along the marked line, except on the proximal side, and proximally-based skin flaps were elevated over the perichondrial plane using a number 15 blade and sharp scissors. Meticulous care must be taken during flap elevation because it is important not to include any perichondrium in the flap; otherwise, interference by the remnant peri-
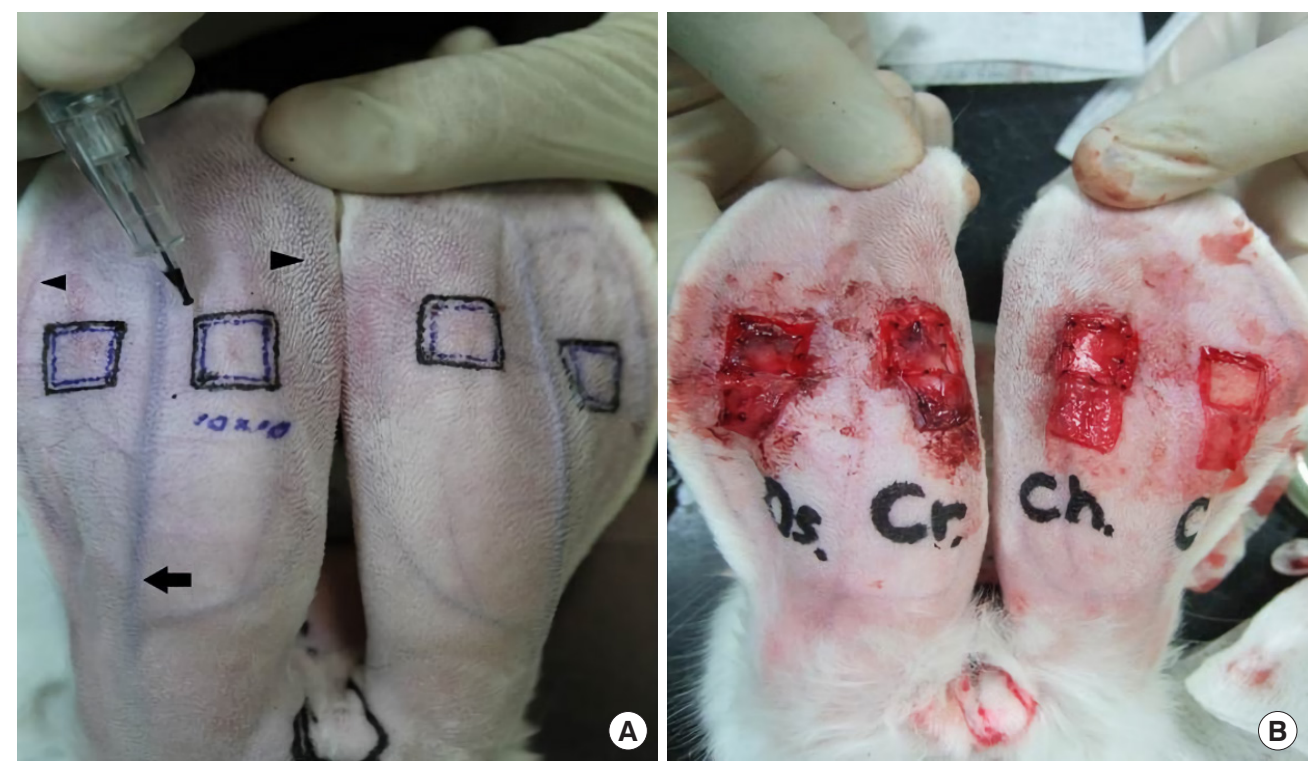

Fig. 1. Surface marking of the flap design on the posterior side of rabbit ears. (A) The inner square $(1.0 \times 1.0 \mathrm{~cm})$ indicates the area of the cartilage defect, and the outer square $(1.2 \times 1.2 \mathrm{~cm})$ indicates the flap margin. The black arrow in the middle indicates the central artery, and the arrowheads indicate the marginal veins bilaterally. (B) Skin flaps were elevated and grafts were used to cover the cartilage defects: from left to right, periosteum taken from tibia, periosteum taken from the cranium, perichondrium, and control. 
chondrium would be an obstacle. A square of ear cartilage measuring $1.0 \times 1.0 \mathrm{~cm}$ was removed, including the perichondrium on both sides.

\section{Preparation of free grafts}

The four cartilage defect sites were randomly assigned and then covered with perichondrium (group 1), periosteum from the calvarium (group 2), or periosteum from the tibia (group 3). In the control group (group 4), no coverage was performed (Fig. 1).

Perichondrium was harvested from the ear cartilage after skin flap elevation (group 1). A careful incision was made on the perichondrium, measuring $1.0 \times 1.0 \mathrm{~cm}$, and then the perichondrium of the posterior side was elevated from the ear cartilage. The free perichondrial graft was re-attached in situ over the ear cartilage defect using 7-0 nylon (Fig. 2). Periosteum was taken from the calvarium (group 2). A midline incision was made on the scalp of the rabbit, followed by supra-pericranial dissection. An area of pericranium measuring $1.0 \times 1.0 \mathrm{~cm}$ was harvested from the parietal side of the cranium, and the donor site was closed using 5-0 nylon. The pericranium was attached to the posterior aspect of the ear cartilage defect in the same manner as in group 1 . The cambium layer faced the anterior side, whereas the fibrous layer faced the posterior flap side (Fig. 2). Periosteum was harvested from the proximal and anteromedial aspects of the right tibia (group 3). The outline of the tibia along with the incision line was designed on the surface, followed by incision and supra-periosteal dissection. An area of periosteum measuring $1.0 \times 1.0 \mathrm{~cm}$ was harvested, and the donor site was closed after meticulous hemostasis. The periosteum was attached to the ear cartilage defect in the same manner as in group 2. In the control group (group 4), the cartilage defect was made by the aforementioned methods, and then the skin flap was closed over the cartilage defect with no additional intervention.

After all graft procedures were completed, the skin flap was closed. The surgical wound was treated with Terramycin antibiotic ointment (Pfizer, New York, NY, USA) without dressing material. Mobic (2 mg/kg; Boehringer Ingelheim, Ingelheim am Rhein, Germany) was administered to alleviate postoperative pain. The results were investigated at 6 weeks after the experiment. All animals were sacrificed through a potassium chloride injection into the marginal vein.

\section{Macroscopic evaluation}

Gross inspection was performed to detect any wound problems such as infection or dehiscence. The characteristics of regenerated tissue were evaluated qualitatively in terms of hardness,

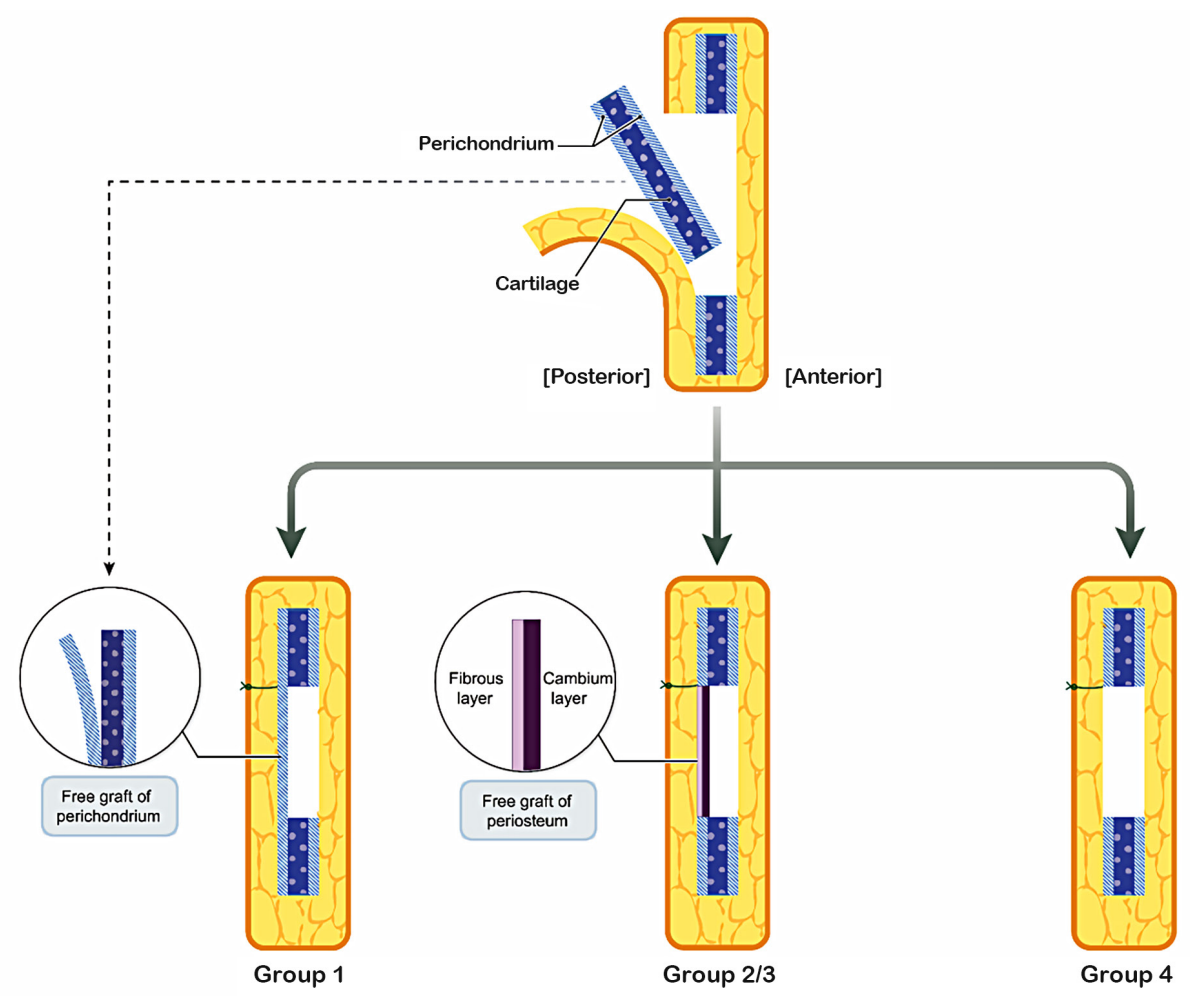

Fig. 2. In group I, perichondrium on the posterior side was elevated from cartilage and re-attached in situ. Cartilage including the perichondrium on the anterior side was removed. In groups 2 and 3, the cambium layer of the periosteum faced toward the cartilage defect area. In group 4 , the cartilage defect was made by the aforementioned method, and the skin flap was closed over the cartilage defect with no additional intervention. Group 1, perichondrium graft; group 2, calvarial periosteum graft; group 3, tibial periosteum graft; group 4, control (no coverage). 
thickness, and the relationship between newly formed tissue and the surrounding normal cartilage. The dimension of the skin flaps was measured to evaluate the effects of fibrosis and wound contraction. The outline of each skin flap was drawn using transparent paper, and the dimensions were calculated using Image (NIH, Bethesda, MD, USA). The wound contraction ratio was estimated by comparing the dimensional changes of the flap to the original flap dimensions.

\section{Microscopic evaluation}

Rectangular specimens measuring approximately $20 \times 5 \mathrm{~mm}$ were harvested in the middle of the flap, including both the defective and the normal side, fixed with $10 \%$ formalin for more than 48 hours, and embedded in paraffin after washing in flowing water for at least 24 hours. Tissue sections ( $5 \mu \mathrm{m}$ thick) were made along the longitudinal axis and then stained with hematoxylin and eosin. The amount of regenerated cartilage or bone tissue was measured using ImageJ and presented as a ratio to the cartilage defect area.

\section{Statistical analysis}

The statistical analysis was performed using SPSS version 19
(IBM Corp., Armonk, NY, USA). A generalized estimating equation was used to evaluate differences in chondrogenesis.

\section{RESULTS}

Of the 12 rabbits in this study, 11 were evaluated 6 weeks after the experiment. One rabbit died as a result of an accident related to anesthesia.

\section{Macroscopic evaluation}

There were no surgical complications such as wound infection, dehiscence, or hematoma. In group 1, the newly formed tissue was similar to the surrounding normal cartilage tissues in terms of thickness and stiffness and fused well with them. In groups 2 and 3 , the regenerated tissue showed a relatively bumpy surface with a yellow to brownish color; it fused with the surrounding normal cartilage, but was thicker and hard, resembling bony tissue. In the control group, the defect area was hollow without sufficient regenerated cartilage tissue (Fig. 3). The wound contraction ratio was higher in group $2(0.32 \pm 0.10)$ and group 3 $(0.38 \pm 0.10)$ than in group $1(0.26 \pm 0.10)$ and group $4(0.29 \pm$ $0.09)$, but the differences were not statistically significant.
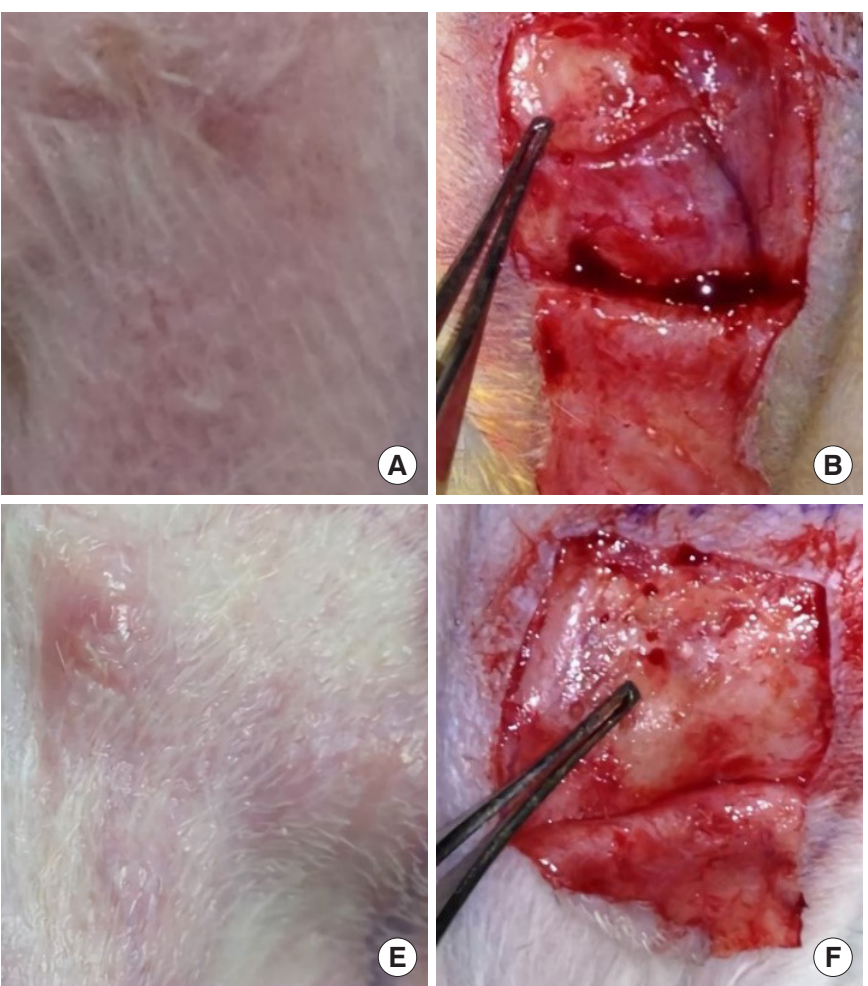
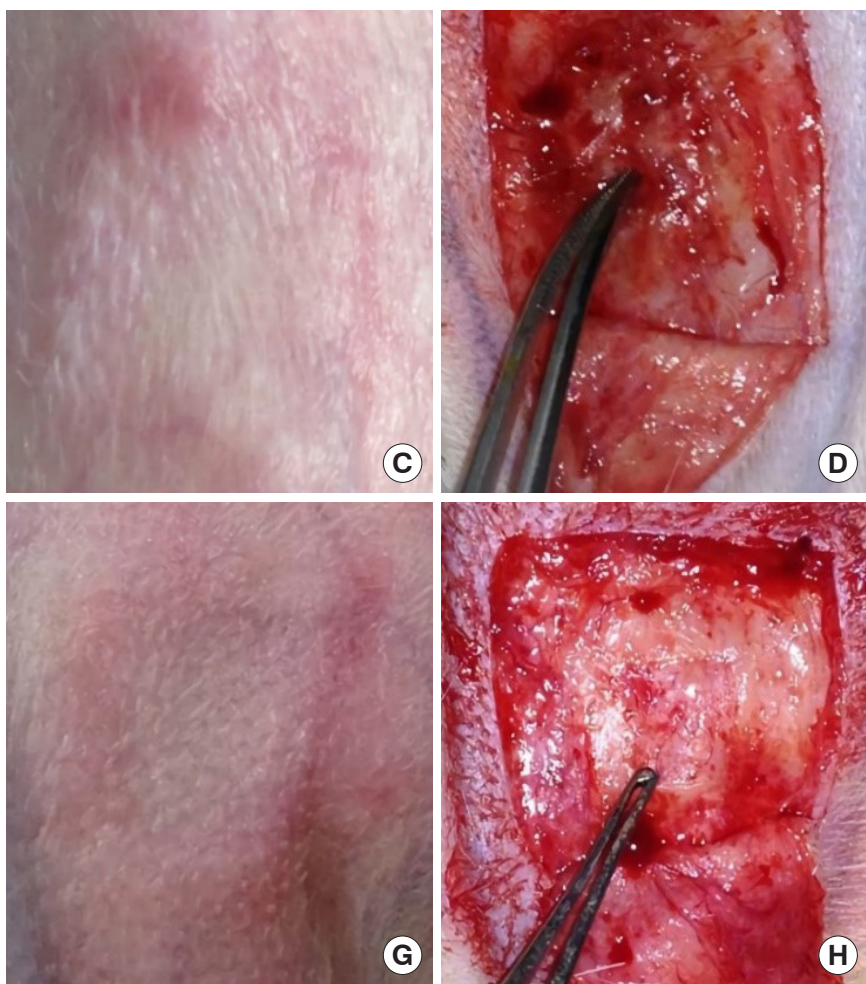

Fig. 3. Macroscopic evaluation. (A, B) In group 1, the newly formed tissue was similar in terms of thickness and stiffness to the surrounding normal cartilage tissues and fused well with them. (C-F) In groups 2 and 3, the regenerated tissue showed a relatively bumpy surface with a yellow to brownish color; it fused with the surrounding normal cartilage, but was thicker and hard, resembling bony tissue. (G, H) In group 4, the defect area was hollow without sufficient regenerated cartilage tissue. Group 1, perichondrium graft; group 2, calvarial periosteum graft; group 3, tibial periosteum graft; group 4, control (no coverage). 


\section{Microscopic evaluation}

Cartilage tissue regeneration was seen in group 1, and the regenerated cartilage fused well with surrounding normal cartilage. However, the thickness of the regenerated cartilage was uneven compared with the normal side, and complete maturation of the cartilage was not observed. The cartilage regeneration ratio in group $1(0.97 \pm 0.60)$ was significantly greater than in group $2(0.10 \pm 0.11)$, group $3(0.08 \pm 0.09)$, and group $4(0.08 \pm$ $0.14)(p=0.004)$. However, there were no statistically significant differences between groups 2,3 , and 4 (Figs. 4, 5).

In groups 2 and 3 , only a small amount of elastic cartilage was regenerated in the vicinity of the normal cartilage tissue. Instead, newly formed osteon was observed in the middle of the defect (Fig. 4). The bone regeneration ratio, calculated by the aforementioned method, was $2.10 \pm 1.97$ in group 2 and $2.92 \pm$ 4.17 in group 3, but no osteogenesis was observed in groups 1 or 4 . There was no statistically significant difference in chondrogenesis or osteogenesis between groups 2 and 3 .

\section{DISCUSSION}

Elastic cartilage is composed of collagen fibers and abundant elastic fibers in a solid matrix. The elastic fibers account for the

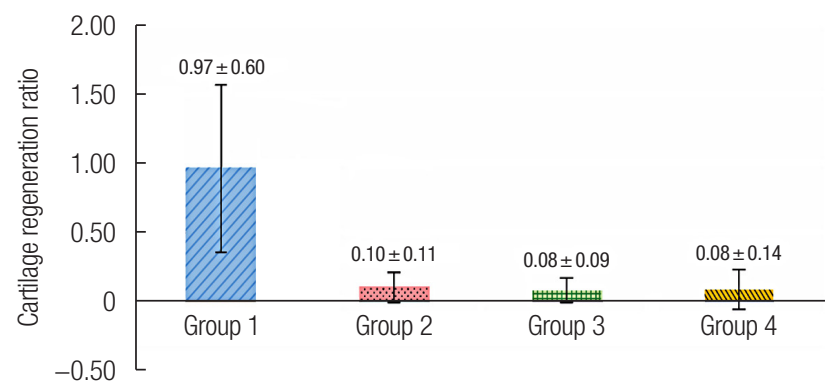

Fig. 5. The cartilage regeneration ratio in group 1 was significantly greater than in groups 2,3 , and $4(p=0.004)$. However, there were no statistically significant differences between groups 2,3 , and 4 . Values are presented as mean \pm SD. Group 1, perichondrium graft; group 2, calvarial periosteum graft; group 3, tibial periosteum graft; group 4, control (no coverage)
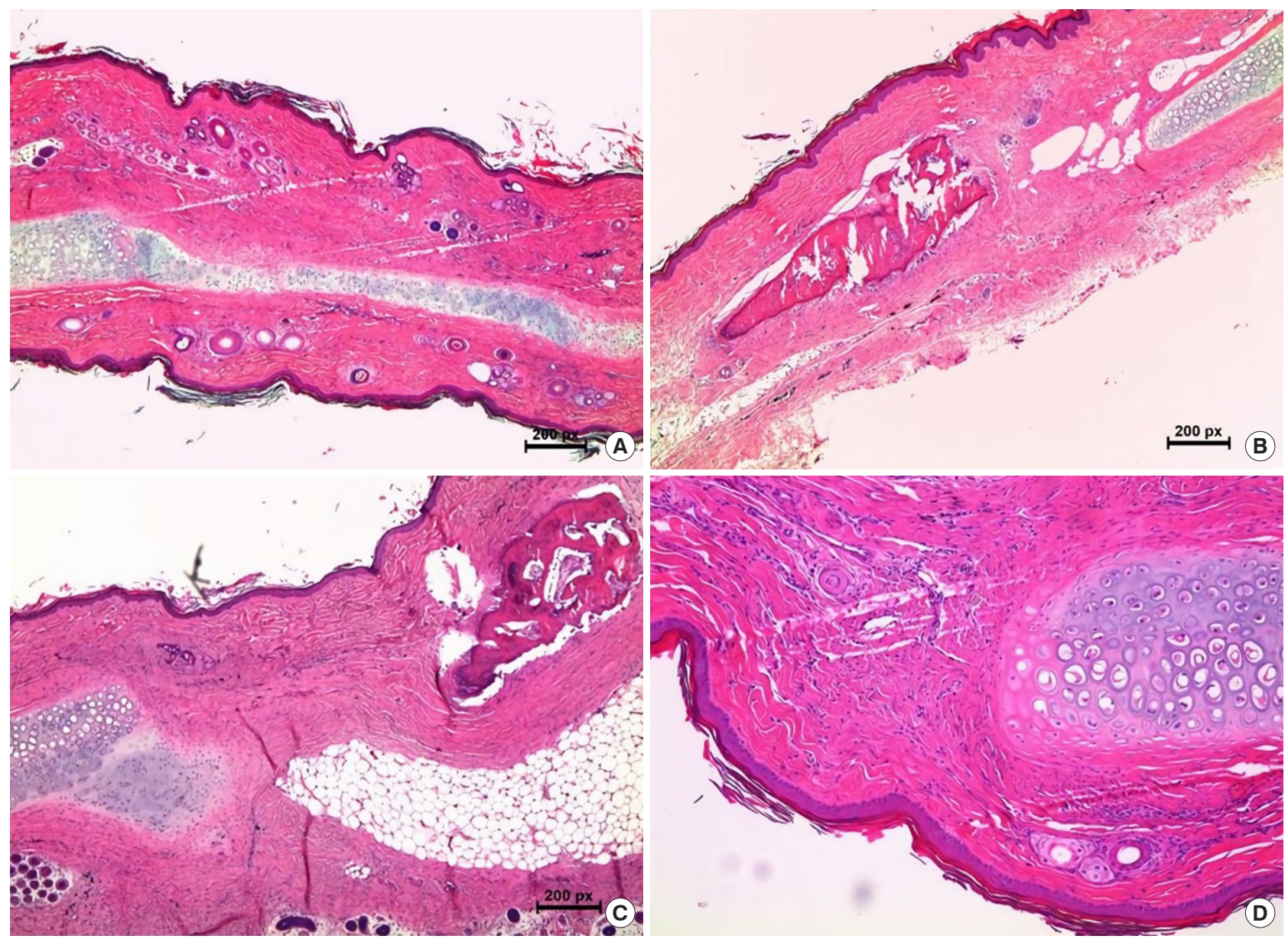

Fig. 4. Microscopic evaluation by hematoxylin and eosin staining. (A) Neo-cartilage tissue regenerated in the cartilage defect area in group 1 $(\mathrm{H} \& \mathrm{E}, \times 40)$. (B, C) There was no chondrogenesis in the periosteal graft groups (H\&E, $\times 40)$. Osteogenesis was observed in group 3 on the anterior side of the periosteal graft, toward which the cambium layer faced. (D) The control group (H\&E, $\times 100$ ). Group 1, perichondrium graft; group 3, tibial periosteum graft. 
flexibility of cartilage, which can endure repeated bending forces and is found in elastic structures that undergo gentle movement, such as the ear, the epiglottis, and the Eustachian tube. The elastic, but durable properties of ear cartilage allow it to be used for various purposes in plastic and reconstructive surgery. However, due to the limited amount of ear cartilage, experiments have been conducted to induce cartilage regeneration using perichondrium or periosteum.

Perichondrium contains multipotent stem cells, which differentiate into chondroblasts $[12,13]$. This technique is used in a limited manner in knee joint cartilage regeneration in a maximally chondrotrophic environment $[10,11]$. Periosteum is also known to have chondrogenic potential when a favorable environment for chondrogenesis is provided. Periosteum is much more abundant than perichondrium, and it is technically easier to harvest periosteum than perichondrium.

Periosteum is composed of two layers; the cambium layer facing the bone cortex contains osteoprogenitors, preosteoblasts, osteoblasts, and other precursor cells that affect bone generation, while the outer fibrous layer is attached to the muscular structure, which transfers contraction forces [14]. Embryologically, periosteum originates from perichondrium, and chondrocyte precursor cells in the cambium layer are thought to be the source of chondrogenesis in a chondrotrophic environment [15].

Previous studies revealed that periosteal grafts had chondrogenic potential equal to that of perichondrial grafts when they were grafted into joint spaces. O'Driscoll et al. [16,17] reported positive results of periosteal grafts on chondrogenesis and argued that lower oxygen pressure, repetitive movement, and young age are important factors, along with other minor contributors to chondrogenesis in periosteal grafts. Ulutas et al. [18] reported comparable cartilage regeneration using periosteal grafts and perichondrial grafts in an ear cartilage defect model.

However, in the present study, periosteal grafts showed poorer chondrogenesis than perichondrial grafts. This result corresponds with previous reports, including recent research showing no significant chondrogenesis of periosteal grafts in the long term despite the concomitant application of chondro-inductive growth factors [19]. Sari et al. [20] compared the chondrogenic potential of perichondrial and periosteal grafts that were wrapped on themselves and transplanted under the abdominis fascia in rabbits, and they did not find any mature cartilage formation from the periosteal grafts. Poussa et al. [21] designed two different models to analyze chondrogenesis from periosteal grafts; one was inserted between the perichondrium and cartilage, which provided an avascular milieu, and the other was transplanted between two skin layers. In the former, neocartilage was observed, and the latter showed bone formation within 1 week without chondrogenesis. Insufficient circulation in the recipient bed was thought to be an important precondition for chondrogenesis from periosteal grafts. In our study, a cartilage defect was created in the head and neck region, which has a different environment from that of the joint space in terms of chondrogenic factors. Synovial fluid in the joint space contributes to an avascular environment while providing growth factors. Our failure to achieve neo-cartilage formation from free periosteal grafts may be attributed to a relatively robust blood supply and the lack of repetitive movements, which are required for optimal chondrogenesis.

Embryologic bone formation occurs in two ways: endochondral ossification in the long bones and intramembranous ossification in the flat bones. Endochondral ossification is generated through a cartilaginous intermediate, while intramembranous ossification is formed without a cartilaginous intermediate [11]. In general, periosteum taken from bones characterized by endochondral ossification, such as the tibia showed superior osteogenicity over those from bones characterized by intramembranous ossification, such as the calvarium or rib [22-24]. However, no studies have been conducted regarding the chondrogenic capacity of periosteum from different donor sites. In the present study, there was no significant difference in chondrogenesis and osteogenesis between periosteal grafts from the calvarium and tibia.

To the best of our knowledge, this is the first report that directly compares the chondrogenic potential of perichondrium, periosteum of intramembranous origin, and periosteum of endochondral origin in an elastic cartilage defect model. In previous research, cartilage regeneration by periosteum was increased when it was treated with basic fibroblast growth factor, transforming growth factor-beta, a mixture of growth factors using platelet-rich plasma, or adipose-derived stem cells [2529]. In vitro expansion of chondrocytes mixed with various growth factors can positively affect cartilage regeneration, and this point could be elucidated in a future study.

Periosteal chondrogenesis was not seen in an ear cartilage defect model. It can be inferred that cartilage regeneration by periosteum can be induced in a well-organized chondrotrophic environment. Future research on chondrocytes and growth factors may contribute to our knowledge of periosteal chondrogenesis.

\section{NOTES}

\section{Conflict of interest}

No potential conflict of interest relevant to this article was reported. 


\section{Ethical approval}

The study was approved by the Institutional Animal Care and Use Committee of Seoul National University Hospital (IACUC No. 13-0164).

\section{ORCID}

Hyokyung Yoo https://orcid.org/0000-0002-9171-0379

Taekeun Yoon https://orcid.org/0000-0002-1070-5165

Hahn-Sol Bae

Min-Suk Kang

Byung Jun Kim https://orcid.org/0000-0001-7950-1219 https://orcid.org/0000-0002-2135-8365 https://orcid.org/0000-0002-6891-3768

\section{Author contribution}

Conceptualization: Byung Jun Kim. Data curation: Hyokyung Yoo, Byung Jun Kim. Formal analysis: Hyokyung Yoo, Min-Suk Kang, Byung Jun Kim. Methodology: Hahn-Sol Bae, Min-Suk Kang, Byung Jun Kim. Project administration: Hyokyung Yoo, T Yoon, Min-Suk Kang. Visualization: Hyokyung Yoo, Taekeun Yoon, Min-Suk Kang. Writing - original draft: Hyokyung Yoo, Taekeun Yoon, Hahn-Sol Bae, Min-Suk Kang. Writing - review \& editing: Hyokyung Yoo, Taekeun Yoon, Hahn-Sol Bae. Investigation: Hahn-Sol Bae. Supervision: Byung Jun Kim. Validation: Byung Jun Kim.

\section{REFERENCES}

1. Hashikawa K, Tahara S, Nakahara M, Sanno T, Hanagaki H, Tsuji Y, et al. Total lower lid support with auricular cartilage graft. Plast Reconstr Surg 2005;115:880-4.

2. Lee Y, Kim J, Lee E. Lengthening of the postoperative short nose: combined use of a gull-wing concha composite graft and a rib costochondral dorsal onlay graft. Plast Reconstr Surg 2000;105:2190-9.

3. Son D, Kwak M, Yun S, Yeo H, Kim J, Han K. Large auricular chondrocutaneous composite graft for nasal alar and columellar reconstruction. Arch Plast Surg 2012;39:323-8.

4. Yoon SH, Kim CS, Oh JW, Lee KC. Optimal harvest and efficient use of septal cartilage in rhinoplasty. Arch Craniofac Surg 2021;22:11-6.

5. Heyner S. The significance of the intercellular matrix in the survival of cartilage allografts. Transplantation 1969;8:666-77.

6. Grosu-Bularda A, Manea C, Lazarescu L, Lascar L. The role of cartilage and bone Allografts in nasal reconstruction. Rom J Rhinol 2016;6:75-82.

7. Ryu YA, Jin M, Kang N. Histological, physical studies after xenograft of porcine ear cartilage. Arch Craniofac Surg 2017;18: $155-61$.

8. Bhardwaj N, Devi D, Mandal BB. Tissue-engineered cartilage: the crossroads of biomaterials, cells and stimulating factors. Macromol Biosci 2015;15:153-82.

9. Jang HU, Kim SY. Biodegradable implants for orbital wall fracture reconstruction. Arch Craniofac Surg 2020;21:99-105.

10. Skoog T, Johansson SH. The formation of articular cartilage from free perichondrial grafts. Plast Reconstr Surg 1976;57:16.

11. Bouwmeester SJ, Beckers JM, Kuijer R, van der Linden AJ, Bulstra SK. Long-term results of rib perichondrial grafts for repair of cartilage defects in the human knee. Int Orthop 1997;21: 313-7.

12. Skoog T, Ohlsen L, Sohn SA. Perichondrial potential for cartilaginous regeneration. Scand J Plast Reconstr Surg 1972;6:1235.

13. Upton J, Sohn SA, Glowacki J. Neocartilage derived from transplanted perichondrium: what is it? Plast Reconstr Surg 1981;68:166-74.

14. Buck DW 2nd, Dumanian GA. Bone biology and physiology: Part I. The fundamentals. Plast Reconstr Surg 2012;129:131420

15. O'Driscoll SW. Articular cartilage regeneration using periosteum. Clin Orthop Relat Res 1999;(367 Suppl):S186-203.

16. O'Driscoll SW, Fitzsimmons JS. The role of periosteum in cartilage repair. Clin Orthop Relat Res 2001;(391 Suppl):S190-207.

17. O'Driscoll SW, Saris DB, Ito Y, Fitzimmons JS. The chondrogenic potential of periosteum decreases with age. J Orthop Res 2001;19:95-103.

18. Ulutas K, Menderes A, Karaca C, Ozkal S. Repair of cartilage defects with periosteal grafts. Br J Plast Surg 2005;58:65-72.

19. Gotterbarm T, Breusch SJ, Vilei SB, Mainil-Varlet P, Richter W, Jung M. No effect of subperiosteal growth factor application on periosteal neo-chondrogenesis in osteoperiosteal bone grafts for osteochondral defect repair. Int Orthop 2013;37:1171-8.

20. Sari A, Tuncer S, Ayhan S, Elmas C, Ozogul C, Latifoglu O. What wrapped perichondrial and periosteal grafts offer as regenerators of new tissue. J Craniofac Surg 2006;17:1137-43.

21. Poussa M, Rubak J, Ritsila V. Differentiation of the osteochondrogenic cells of the periosteum in chondrotrophic environment. Acta Orthop Scand 1981;52:235-9.

22. Uddstromer L. The osteogenic capacity of tubular and membranous bone periosteum: a qualitative and quantitative experimental study in growing rabbits. Scand J Plast Reconstr Surg 1978;12:195-205.

23. Iuchi T, Kusuhara $H$, Ueda $Y$, Morotomi T, Isogai N. Influence of periosteum location on the bone and cartilage in tissue-engineered phalanx. J Hand Surg Am 2020 Jan;45(1):62.e1-10.

24. Fujii T, Ueno T, Kagawa T, Sakata Y, Sugahara T. Comparison of bone formation ingrafted periosteum harvested from tibia 
and calvaria. Microsc Res Tech 2006;69:580-4.

25. Fujisato T, Sajiki T, Liu Q, Ikada Y. Effect of basic fibroblast growth factor on cartilage regeneration in chondrocyte-seeded collagen sponge scaffold. Biomaterials 1996;17:155-62.

26. Zhu Y, Yuan M, Meng HY, Wang AY, Guo QY, Wang Y, et al. Basic science and clinical application of platelet-rich plasma for cartilage defects and osteoarthritis: a review. Osteoarthritis Cartilage 2013;21:1627-37.

27. Van Pham P, Bui KH, Ngo DQ, Vu NB, Truong NH, Phan NL, et al. Activated platelet-rich plasma improves adipose-derived stem cell transplantation efficiency in injured articular cartilage. Stem Cell Res Ther 2013;4:91.

28. Kenmochi M. Clinical outcomes following injections of leukocyte-rich platelet-rich plasma in osteoarthritis patients. J Orthop 2019;18:143-9.

29. Vinod E, Amirtham SM, Kachroo U, Goyal A, Ozbey O, James JV, et al. Articular chondroprogenitors in platelet rich plasma for treatment of osteoarthritis and osteochondral defects in a rabbit knee model. Knee 2021;30:51-62. 